\title{
A study protocol for a randomised controlled trial to evaluate the effectiveness of a dog-facilitated physical activity minimal intervention on young children's physical activity, health and development: the PLAYCE PAWS trial
}

\author{
Michelle $\mathrm{Ng}^{1 *}$ (D) Elizabeth Wenden ${ }^{1,2}$, Leanne Lester ${ }^{3}$, Carri Westgarth ${ }^{4}$ and Hayley Christian ${ }^{1,2}$
}

\begin{abstract}
Background: Pet ownership brings many health benefits to individuals. In children developmental benefits can extend to improved self-esteem, better social competence and decreased loneliness. The majority of households with children own a dog, however only a small proportion of children gain the benefits of dog ownership through dog walking and play. There are few intervention studies investigating the impact of dog-facilitated physical activity in children. The PLAYCE PAWS study aims to test a minimal-contact intervention through the use of mobile health ("mhealth") strategies, i.e. text (SMS) messages, to parents to encourage their children to walk and play with their dog more, and evaluate the impact on children's overall physical activity and development.

Methods/design: The PLAYCE PAWS intervention study will target parents in dog-owning families with children aged 5 to 8 years in Perth, Western Australia. Approximately 150 dog-owning parents and children will be randomly allocated into either one of two intervention groups or a 'usual care' control group. The first intervention group will receive SMS messages over 4 weeks to encourage and prompt parents to undertake dog walking and dog play with their child. The second intervention group will receive the same text messages, plus a dog pedometer and personalised 'dog steps' diary for their child to complete. Parent-reported outcome measures include changes in children's dog walking and play, overall physical activity, socio-emotional development, self-regulation, self-esteem, empathy, and level of attachment to their dog.

(Continued on next page)
\end{abstract}

* Correspondence: michelle.ng@telethonkids.org.au

'Telethon Kids Institute, University of Western Australia, Crawley, WA, Australia

Full list of author information is available at the end of the article

(c) The Author(s). 2020 Open Access This article is licensed under a Creative Commons Attribution 4.0 International License, which permits use, sharing, adaptation, distribution and reproduction in any medium or format, as long as you give appropriate credit to the original author(s) and the source, provide a link to the Creative Commons licence, and indicate if changes were made. The images or other third party material in this article are included in the article's Creative Commons licence, unless indicated otherwise in a credit line to the material. If material is not included in the article's Creative Commons licence and your intended use is not permitted by statutory regulation or exceeds the permitted use, you will need to obtain permission directly from the copyright holder. To view a copy of this licence, visit http://creativecommons.org/licenses/by/4.0/ The Creative Commons Public Domain Dedication waiver (http://creativecommons.org/publicdomain/zero/1.0/) applies to the data made available in this article, unless otherwise stated in a credit line to the data. 
(Continued from previous page)

Discussion: The PLAYCE PAWS study appears to be the first to examine the effectiveness of a low-cost, mhealth intervention for increasing young children's physical activity through dog walking and play. Given the high prevalence of dogs as family pets, this study presents a valuable opportunity to investigate if mHealth interventions encourage children to walk and play with their dog more, and if there are any associated impact on children's overall physical activity and socio-emotional well-being. If effective, a larger trial or program could be implemented at low-cost and with wide reach in the community.

Trial registration: ANZCTR, ACTRN12620000288921. Registered 4th March 2020 - Retrospectively registered.

Keywords: Children, Dog, Dog walking, Dog play, mHealth, Development, Physical activity

\section{Background}

\section{Dog ownership and children's physical activity benefits}

Pet ownership is associated with a number of physical, mental and emotional health benefits [1-3]. There is a growing body of research on the physical activity benefits of dog ownership. In adults, dog ownership is associated with higher physical activity levels and increased likelihood of meeting physical activity recommendations $[4,5]$. Fewer studies have been conducted on children but overall they show that children from dog-owning families accumulate more physical activity [6-8], and are more likely to meet physical activity recommendations $[6,9]$. Furthermore, children who walk their dog, play in the street and yard more and are more independently mobile compared with children who don't walk their dog [10].

A large proportion of households with children own a dog: in the U.S. and Australia $50-70 \%$ of households with children have a dog $[8,11,12]$, and in the UK approximately $22-24 \%$ of households own a dog $[13,14]$ with dogs more common in households with children [14]. Despite this high level of dog ownership, many dog-owning children do not engage in any dogfacilitated physical activity, in particular dog walking [6, $7,15]$. An Australian study found only $23 \%$ of $5-6$ year olds ever walked their dog, walking on average 1.7 times per week with their dog [8]. Increased walking resulting from dog ownership may be an overlooked mechanism for increasing children's physical activity [6].

\section{Dog ownership and children's development}

Dog ownership has been associated with other psychological, social and developmental benefits in children. These include better social-emotional development [11], improved self-esteem, autonomy, empathy, trust and self-confidence, increased feelings of safety, social competence [16-18], and family cohesion [19]. For example, a longitudinal study of more than 4000 Australian children aged 5 to 7 years found that dog ownership was associated with lower abnormal scores on any of the Strengths and Difficulties Questionnaire scales (a measure of a child's social-emotional development) [11]. One of the mechanisms through which the benefits of pets may be conferred to children could be through the level of attachment to a pet $[20,21]$. There is some evidence to suggest that children who are more attached to their dogs spend more time being active with them [15, 22].

Key factors influencing how dog ownership facilitates improved developmental outcomes for children relates to the quality of the child-dog relationship. This includes factors such as the bond and attachment a child has to the family dog $[16,22]$ as well as the time spent interacting with the family dog. Since dog-facilitated play and family dog walks enables children to spend more time interacting and bonding with their dog, it may be a key mechanism for facilitating increased attachment and developmental benefits such as improved self-esteem, self-regulation and empathy. In addition, regular physical activity has been shown to beneficial for children's socio-emotional development (such as enhanced social skills and emotional intelligence), mental health (such as reduced depression and anxiety problems) and better sleep [23-25]. Based on key concepts from relationship psychology [26] and attachment theory [27], a theoretical model of the proposed relationships between dog ownership, dog-facilitated physical activity and developmental outcomes in young children is shown in Fig. 1.

\section{Intervention studies to increase dog-facilitated physical activity}

Intervention studies to promote dog-facilitated physical activity have been few, and only one has targeted children. The Children, Parents and Pets Exercising Together (CPET) pilot intervention utilised various strategies (in-person visits, phone calls and text messaging) over 10 weeks to encourage children $(n=28$, 9-11 year olds) to play/walk their dog [28]. While no significant differences were found between the intervention and control group, mostly due to the small sample size, families found the intervention to be acceptable and feasible.

Two studies in adults have also utilised minimal intervention strategies involving mobile health (mHealth) to increase physical activity through dog walking $[29,30]$. 




Fig. 1 Theoretical model of the relationship between family dog ownership, dog-facilitated and development in young children

A U.S. study $(n=102)$ utilised online social networks to promote weekly neighbourhood dog walks [30] while another $(n=105)$ trialled weekly email messages to promote the benefits and reduce barriers to dog walking [29]. Both studies found mHealth interventions to be effective at increasing dog walking amongst participants in the intervention compared with the control group at follow-up. However, both studies were pilot interventions and thus were limited by small sample sizes.

Physical activity interventions that are relatively low cost, require little face-to-face interaction between participants and those delivering the intervention, and encourage participant involvement in self-selected physical activity may have better long term success [31] compared with traditional interventions which involve closely supervised intensive, and expensive programs $[31,32]$. Thus, larger trials with more rigorous research methodology (i.e., random sampling; adjustment for confounders; use of context-specific measures) are needed to determine the best strategies for increasing children's physical activity through active play and walking with the family dog $[16,33]$. In addition, it is important to understand if increased interaction and bonding with the family dog facilitated by increased dog play and family dog walking, leads to other developmental benefits for children.

The aim of this study is to determine if a dog-facilitated physical activity minimal intervention: a) increases the amount of active play, family dog walking and overall physical activity levels; b) increases the amount of time children spend interacting with their family dog and their level of attachment to the dog; and c) improves child development outcomes. We hypothesize that strategies to increase children's active play and walking with the family dog will improve children's physical activity levels and development.

\section{Methods/ design}

\section{Study sample, recruitment, inclusion and exclusion} criteria

This is a sub-study of the Play Spaces \& Environments for Children's Physical Activity (PLAYCE) study, which was a cross-sectional observational study conducted from 2015 to 2018 [34]. The PLAYCE study investigated the influence of the home, neighbourhood and early childhood education and care (ECEC) environment on the physical activity levels of children attending ECEC [34]. Participants included 1596 children aged between 2 and 5 years from 104 ECECs in the Perth metropolitan area, Western Australia [35]. Children are now being followed up as they enter full-time school. Specific details of the PLAYCE study's methods have been published elsewhere [34].

To reach the target sample size $(n=150)$, participants will be recruited from both the PLAYCE cohort [34], and the general community by multiple strategies including advertising in print (newspapers, school and professional association newsletters) and social media (Facebook and Twitter), crowdsourcing (via institutional websites), market research and through snowball sampling. Parents will be invited to participate in the study by filling in an online expression-of-interest form or by contacting the study team directly. The study team will follow-up interested parents to confirm their eligibility to participate. Parents with children between 5 to 8 years and who have a family $\operatorname{dog}(\mathrm{s})$ that is well socialised with the child and other people will be eligible to participate. Children with a recognised disability (physical, emotional/behavioural or intellectual) that would affect participation in physical activity will be ineligible.

\section{Sample size considerations}

This project is an exploratory pilot trial, intended in part to inform future sample size calculations. This study aims to recruit 50 participants into each group (total $n=150$ ). Based on preliminary analysis of PLAYCE data [35] and the work of Morrison et al. 2013 (CPET study, 2013) [36], this study will have more than $80 \%$ power to detect a one-unit difference in the pre-post change in the number of times per week children play or walk with their dog between the intervention and control groups. There are no comparable intervention data in this young group of children to accurately inform a power calculation. However, based on data from the CPET study [36], and the PLAYCE study [35], it is expected that the response within each subject group will be normally distributed with a standard deviation of 1.5. If the true difference in the experimental and control means is one 
additional dog walk or play session with the dog per week, we will be able to reject the null hypothesis that the population means of the experimental and control groups are equal with a probability (power) of 0.91 . The Type I error probability associated with this test of the null hypothesis is 0.05 . This study is also sufficiently powered to detect a difference in children's physical activity, and development outcomes between intervention and control groups, since the outcome measures are context specific (e.g., dog walking/play and home-based outdoor play) compared with the CPET study (overall physical activity) [36].

\section{Design and randomisation}

This is a block randomised controlled study with two intervention groups and a 'usual care' control group (see Fig. 2) with equal sample sizes in each group $(n=50 /$ group; 3 groups; total $=150$ ). . Baseline measurements will be collected after eligible participants are screened and recruited (T0). Participants will then be randomly allocated in staggered blocks into one of the three groups. Follow-up measurements will be collected at one-month (F1) and three-months post-intervention (F2) (see Table 1).

\section{Intervention and control groups}

Physical activity -based minimal intervention strategies will be tested over a four-week period, consisting of mHealth SMS message prompts to parents and a dog pedometer and personalised dog steps diary for children. The study consists of two intervention groups: the 'SMS only group' and 'SMS and dog pedometer group' and a 'usual care' control group. Both intervention groups will be sent a personalised mobile phone SMS message three times a week (two sent during the work week and one sent on the weekend) to motivate and encourage parents to support their child to either walk and/or play with their dog each day. Examples include: "Has $<\mathrm{dog}>$ been outdoors today? Spring is a great time to go for a walk with $<\operatorname{dog}>$ and $<$ child $>$ "; "Physical activity can make us

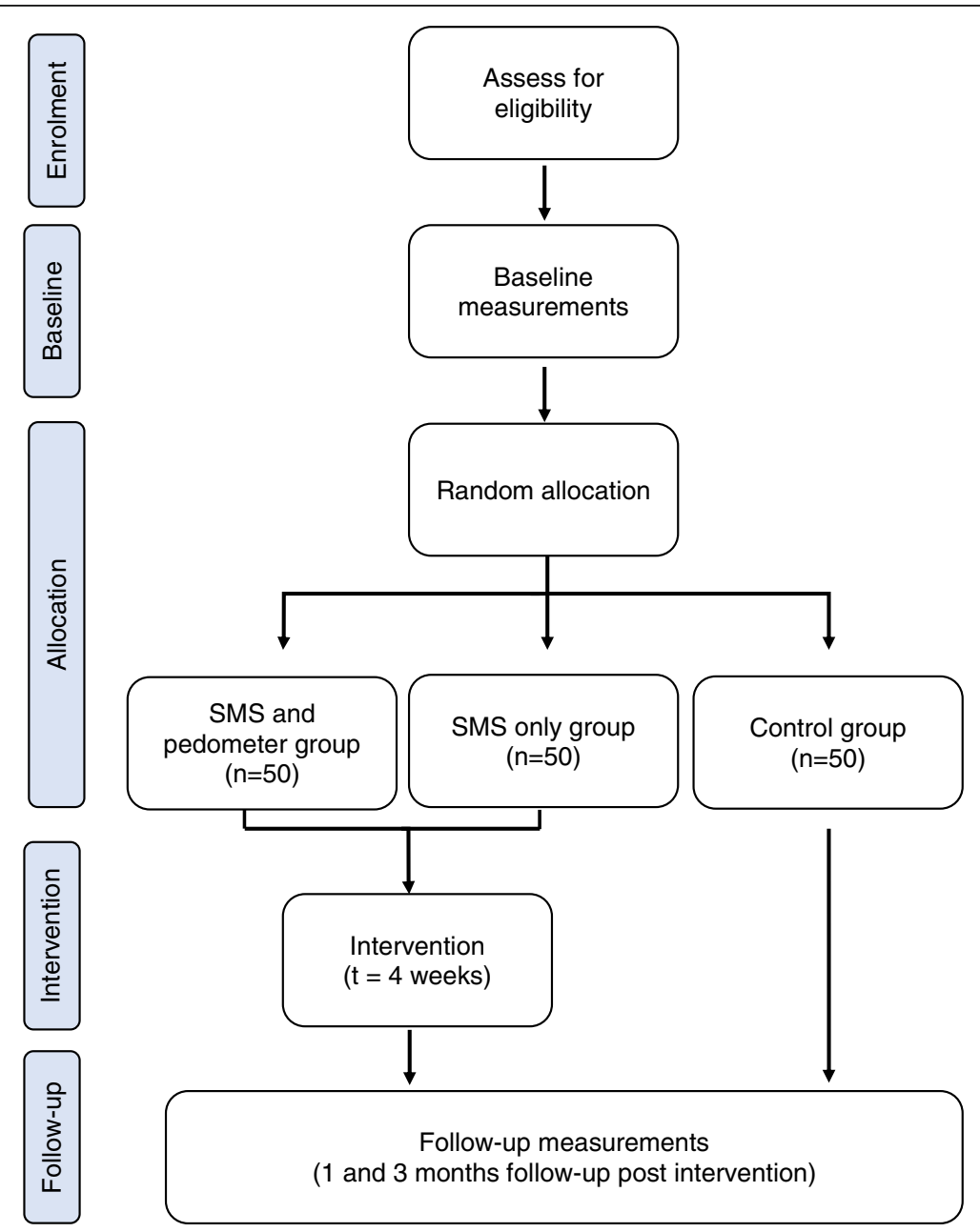

Fig. 2 Overview of study design and recruitment process for the PLAYCE PAWS trial 
Table 1 SPIRIT flow diagram of the PLAYCE PAWS trial protocol

\begin{tabular}{|c|c|c|c|c|c|}
\hline \multirow[t]{2}{*}{ Time point } & $-\mathrm{T} 1$ & T0 & $\mathrm{T1}$ & F1 & F2 \\
\hline & Enrolment & $\begin{array}{l}\text { Baseline/ } \\
\text { Allocation }\end{array}$ & Intervention & $\begin{array}{l}\text { Follow- } \\
\text { up } \\
\text { 1-month }\end{array}$ & $\begin{array}{l}\text { Follow- } \\
\text { up } \\
\text { 3-months }\end{array}$ \\
\hline \multicolumn{6}{|l|}{ Enrolment } \\
\hline Eligibility screening & $x$ & & & & \\
\hline Informed consent & $x$ & & & & \\
\hline Allocation & & $x$ & & & \\
\hline \multicolumn{6}{|l|}{ Intervention } \\
\hline SMS only intervention group & & $x$ & $x$ & $x$ & $x$ \\
\hline SMS and pedometer intervention group & & $x$ & $x$ & $x$ & $x$ \\
\hline Usual care group (Control) & & $x$ & & $x$ & $x$ \\
\hline \multicolumn{6}{|l|}{ Assessment (parent report) ${ }^{\mathrm{a}}$} \\
\hline \multicolumn{6}{|l|}{ Physical activity variables } \\
\hline Structured physical activity & & $x$ & & $x$ & $x$ \\
\hline $\begin{array}{l}\text { Unstructured physical activity (including dog play and family dog } \\
\text { walking) }\end{array}$ & & $x$ & & $x$ & $x$ \\
\hline Outdoor play time & & $x$ & & $x$ & $x$ \\
\hline \multicolumn{6}{|l|}{ Development variables } \\
\hline Social-emotional development & & $x$ & & $x$ & $x$ \\
\hline Self-esteem & & $x$ & & $x$ & $x$ \\
\hline Empathy & & $x$ & & $x$ & $x$ \\
\hline Self-regulation & & $x$ & & $x$ & $x$ \\
\hline Dog attachment variables & & $x$ & & $x$ & $x$ \\
\hline Socio-demographic information & & $x$ & & $x$ & $x$ \\
\hline
\end{tabular}

${ }^{\text {aSee }}$ 'Methods: Outcome measures' section below for full details of variables.

feel cheerful. What activity will you, $<$ child $>$ and $<$ dog $>$ do today?". The 'SMS and dog pedometer' group will also receive a Yamax SW200 pedometer for their dog's collar and a personalised dog steps diary children can use to record the number of pedometer steps their dog does during play or walking. We hypothesize that the use of the dog pedometer and personalised dog steps diary will provide additional encouragement and motivation for parents to take their child and dog for a walk or play each day, and may result in a larger effect compared with the SMS only intervention group and control group.

In order to help facilitate dog walking and dog play, participants in both intervention groups will be provided with information about dog friendly parks, trails and beaches; games for children to play with their dog; and tips about how children can safely interact with their dog. Participants in the control group will be asked to continue their normal routine without any contact from researchers. To ensure fair access to any beneficial outcomes of the project, the control group will receive the same resources as the intervention groups at the end of the study (i.e., information about dog friendly parks, trails and beaches; games for children to play with their dog; and tips about how children can safely interact with their dog).

\section{Outcome measures}

Parents will complete three online surveys to measure changes in children's dog play, family dog walking, overall physical activity, self-regulation, self-esteem, empathy, and level of attachment to their dog.

\section{Change in children's physical activity}

Existing items from the PLAYCE parent-report survey [34] and adapted from the Healthy Active Preschool Years Study (HAPPY Study) [37] will be used to measure children's frequency of active play with their dog, family dog walking, structured (e.g., swimming, gymnastics, football) and unstructured (e.g., playing in the yard, riding bike, active play with toys) physical activity (response scale for all items: 'never/rarely', less than once/week, 34 times/week, 5-6 times /week, daily). The reliability of these items is sound (e.g., unstructured physical activity items intraclass correlation $(\mathrm{ICC})=0.63$; structured physical activity items $\mathrm{ICC}=0.70$ ) [37]. Outdoor play time will be measured using an established tool [38] 
where parents report the amount of time their child spends outdoors $(0,1-15,16-30,31-60,>60 \mathrm{mins})$ across three periods of the day (morning to before lunch; after lunch to $6 \mathrm{pm} ; 6 \mathrm{pm}$ to bedtime). These items have previously been validated against young children's accelerometer data $(r=0.33, p<0.001)$ [38].

\section{Change in children's development}

Social-emotional development will be measured using the parent-report Strengths and Difficulties Questionnaire (SDQ) [39]. The SDQ is a validated and commonly used 25-item (scale: 'not true', 'somewhat true', 'certainly true') instrument that measures the social and emotional well-being of children aged $2-17$ years. Items are combined to create five sub-scales (emotional symptoms, conduct problems, hyperactivity-inattention, peer relationship problems, pro-social behaviour) and a total difficulties score [39]. The parent-report version of the SDQ has satisfactory reliability [40].

Self-esteem will be measured using the Rosenberg SelfEsteem Scale [41]. This instrument includes ten items (5 point scale: strongly disagree to strongly agree) measuring both positive and negative feelings that a child has [41]. The scale has good reliability; test-retest correlations $0.82-0.88$, and Cronbach's alpha for various samples range 0.77-0.88 [42, 43].

Empathy will be measured using the Young Children's Empathy Measure [44]. Parents report their child's ability to identify sadness, fear, anger and happiness in four scenarios: sadness (a child has just lost its best friend), fear (a child is chased by a big, nasty monster), anger (a child really wants to go out but is not allowed) and happiness (a child is going to its most favorite park to play'. The response scale options are 'exact match to the intended emotion'; 'similar emotion'; 'some emotion'; 'non-emotional response'; 'no response'. The measure has acceptable internal reliability (Cronbach alpha 0.69) [44]. To measure the child's empathy towards dogs, the questions will be repeated with 'dog' as the subject of the four statements [44].

Self-regulation will be measured using the Modified Child Problem Behaviour Checklist from the Fast Track Project [45]. This checklist has been generated using items from the Child Behaviour Checklist [46], the Revised Behaviour Problem Checklist [47] and other items developed by the program's investigators (for further details, see Lochman \& The Conduct Problem Prevention Research Group [48]). The checklist includes 20 items measuring the frequency of child externalizing behavior problems ('none of the time, 'some of the time', 'most of the time', 'all of the time'), and has demonstrated high internal consistency $(\alpha=0.87)$ [48].

The level of attachment the child has to their family dog will be measured using items from the Dogs and
Physical Activity Tool (DAPA Tool) [49]. This Tool has been shown to be a reliable tool for measuring adultreported levels of attachment to their dogs, with good to excellent ICC values (ICC $=0.65-0.92)$ [49]. The Inclusion of Other in the Self (IOS) Scale by Aron and colleagues [50], which measures how close a respondent feels with another person will also be used and has been previously modified to measure how close the child feels about 'dog' instead of 'another person or group'. [51, 52].

\section{Potential confounders}

Potential confounders will include parent and child socio-demographic factors (e.g., age, gender, ethnicity, socio-economic status [53]), child's sleep [53] and preference for physical activity [54]. We will adjust for baseline levels of children's dog play and family dog walking. To account for any seasonal variations in children's physical activity-related variables we will also collect information on weather conditions from the Australian Bureau of Meteorology [55].

\section{Participant response and retention}

In order to ensure optimum response rates a number of response and retention methods will be employed. A SMS reminder message will be sent to participants 1 week before the survey is due for completion, followed by an email containing a link to the online survey the week the survey is due for completion. A reminder email will be sent to participants who have not completed the survey 1 week later. If further prompting to complete the survey is required, a follow-up phone call and /or SMS message reminder, as well as a paper copy of the survey, will be posted to participants with a reply-paid envelope after 2 weeks. To encourage surveys to be completed in a timely manner, participants who complete the survey within a four-week period will be eligible to go into the draw to win a \$100 AUD gift card. Incentives, including packets of dog treats and a frisbee, will be given at different stages of the study (e.g., completion of first and final follow-up surveys) to encourage the timely completion and return of study materials and equipment (pedometers and diaries). At the end of the study, all participants will be provided with a report of the study key findings.

\section{Statistical analysis}

Data will be entered electronically on a secure file storage system and password protected. Data will be anonymised by assigning a unique identification number to each participant.

Analyses will involve repeated measures linear models adjusting for relevant confounding variables. The dependent variables will be mean change in 1) frequency of dog play and walking the dog, 2) overall physical 
activity levels, and 3) development (social-emotional development as measured by the SDQ, self-esteem, empathy, self-regulation, child-dog attachment scores) between pre- and post-intervention time points. The independent variable will be the group (intervention group 1 , intervention group 2 or control) participants are assigned to. Between group differences in baseline variables will be examined. Analyses will be based on intention to treat. Effect modifiers and confounders such as baseline levels of dog play, dog walking frequency and socio-demographic factors will be examined and adjusted for where necessary.

\section{Dissemination of project findings}

A study report presenting overall findings of the research will be prepared for all participants and stakeholders. Peer-reviewed publications will allow the results to be disseminated to the scientific community.

\section{Discussion}

The aim of the current study is to test if a dog-facilitated physical activity minimal intervention increases the amount of time young children spend interacting and bonding with their dog, thereby improving physical activity levels and developmental outcomes.

Of the studies examining the relationship between dog walking and physical activity in children and adolescents $[6,7,15,22,56-59]$, only one has been conducted with younger children (less than 9 years old) [8]. Most studies have not considered the types of dog-facilitated physical activity young children are likely to engage in (e.g. play with family dog, family dog walking) [7]. Importantly, there is a lack of intervention studies to determine if dog-facilitated physical activity can increase young children's overall physical activity levels. Our study aims to address this evidence gap.

The current study also seeks to investigate if children who spend more time engaged in play and walking with the family dog improve their bond and attachment with their dog, which may directly or indirectly help facilitate aspects of a child's development. There is some evidence suggesting that children are more likely to report walking their dog if they have a high attachment to it [60]. There is also recent strong evidence that dog ownership (and dog-facilitated physical activity) is associated with improved social-emotional development in young children. For example, a recent Australian study of 1646 children aged 2 to 5 years found that dog ownership was associated with reduced likelihood of conduct problems, peer problems, and increased likelihood of prosocial behaviour compared with children without a dog [61]. Another small study of 27 8-12 year olds found children's behaviour improved (less naughty, more cooperative) 1 month after acquiring a dog compared to non-dog owners, however no significant differences were observed at the 6- and 12- month follow-up [62]. Thus, there is a need for larger intervention studies to investigate whether increasing dog facilitated play and walking has a positive impact on young children's development.

Many traditional physical activity interventions tend to be labour intensive, costly, with short term effects lost once support has been withdrawn [63]. It has been suggested that a 'paradigm shift' is required in physical activity interventions, moving to encouraging more 'purposeful' activity such as walking the dog or walking or biking to destinations [63]. In addition, even the provision of single contact education (e.g. providing education material outlining the benefits of dog walking and dog walking tips) of the importance of dog walking has been shown to be beneficial in increasing adult physical activity levels [64]. Past adult dog walking intervention studies have shown promise but lack evidence of their ability for scale up and application to other population groups such as children [33].

This study utilises simple mHealth strategies which can be easily scaled up and incorporated into mass media campaigns targeting population-level walking across diverse community settings. If shown to be effective, the PLAYCE PAWS intervention may provide an opportunistic, low cost, wide reaching strategy for increasing children's physical activity and improving developmental outcomes.

\section{Abbreviations \\ mHealth: Mobile health; PLAYCE: Play Spaces \& Environments for Children's Physical Activity; SDQ: Strengths and Difficulties Questionnaire; SMS messages: Text messages; SPIRIT: Standard Protocol Items: Recommendations} for Interventional Trials

\section{Acknowledgements}

Hayley Christian is supported by a National Health Foundation Future Leader Fellowship (\#100794 and 102549). Elizabeth Wenden is supported by an Australian Research Training Program Scholarship. Dog treat packets are sponsored by Mars Petcare Australia.

\section{Authors' contributions}

$\mathrm{HC}$ conceived and designed the study. HC, EW, LL, CW and MN contributed to the study methods. MN, HC and EW drafted the manuscript and revised it critically at each stage. All authors contributed to the drafting of manuscript; and read and approved the final manuscript.

\section{Funding}

Human-Animal Bond Research Institute (HABRI) Grant no. HAB17-017 and the Western Australian Health Promotion Foundation (Healthway - \#24219). The funding bodies will not be involved in data analysis or interpretation or manuscript writing.

\section{Availability of data and materials}

The datasets used and/or to be analysed during the current study, in addition to data collection forms are available from the corresponding author on reasonable request.

\section{Ethics approval and consent to participate}

All parents will be provided with a participant information sheet and will provide written informed consent for their and their child's participation in the study. Participants are free to withdraw from the study at any point 
without giving reason. The study has the approval of the University of Western Australia Human Research Ethics Committee (RA/4/1/7417). As the study involves children interacting with their family dog, additional screening questions will be asked to ensure child safety and suitability of the family dog to participate in the study. Examples of screening questions include 'Does the dog have any health/medical/behavioural problems that may prevent them from going for walks or playing with the child' and 'Does your dog display aggressive behaviour (such as growling or snapping) when toys are taken away by a family member'. These screening questions have been drafted in consultation with co-author Westgarth, who is a full member of the UK Association of Pet Behaviour Counsellors. It is not the intention of the study for children to walk or play with their dog alone and adult supervision will be required at all times when the child is interacting with their dog. This will be highlighted in the participant information sheet.

\section{Consent for publication}

Not applicable.

\section{Competing interests}

The authors declare that they have no competing interests.

\section{Author details}

${ }^{1}$ Telethon Kids Institute, University of Western Australia, Crawley, WA, Australia. ${ }^{2}$ School of Population and Global Health, University of Western Australia, Perth, WA, Australia. ${ }^{3}$ School of Human Sciences, University of Western Australia, Crawley, WA, Australia. ${ }^{4}$ Department of Livestock and One Health, Institute of Infection, Veterinary and Ecological Sciences, University of Liverpool, Leahurst Campus, Neston, UK.

Received: 18 November 2020 Accepted: 9 December 2020

Published online: 06 January 2021

\section{References}

1. Allen DT. Effects of dogs on human health. J Am Vet Med Assos. 1997;210 1136-9.

2. Herzog $H$. The impact of pets on human health and psychological wellbeing: fact, fiction, or hypothesis? Curr Dir Psychol Sci. 2011;20(4):236-9.

3. McNicholas J, Gilbey A, Rennie A, et al. Pet ownership and human health: a brief review of evidence and issues. BMJ. 2005;331(7527):1252-4.

4. Westgarth $\mathrm{C}$, Christley R, Christian $\mathrm{H}$. How might we increase physical activity through dog walking?: a comprehensive review of dog walking correlates. Int J Behav Nutr Phys Act. 2014;11:83.

5. Christian H, Westgarth C, Bauman A, Richards EA, Rhodes R, Evenson K, et al. Dog ownership and physical activity: a review of the evidence. J Phys Act Health. 2013;10:750-9.

6. Martin K, Wood L, Christian H, et al. Not just "a walking the dog": dog walking and pet play and their association with recommended physical activity among adolescents. Am J Health Promot. 2015;29:353-6.

7. Owen G, Nightingale C, Rudnicka A, Ekelund U, McMinn A, van Slujs E, et al. Family dog ownership and levels of physical activity in childhood: findings from the child heart and health study in England. Am J Public Health. 2010; 100:1669-71.

8. Salmon J, Timperio A, Chu B, Veitch J. Dog ownership, dog walking, and children's and parents' physical activity. Res Q Exerc Sport. 2010;81:264-71.

9. Christian H, Trapp G, Lauritsen C, Wright K, Giles-Corti B. Understanding the relationship between dog ownership and children's physical activity and sedentary behaviour. Pediatr Obes. 2013:8(5):392-403.

10. Christian H, Trapp G, Villanueva K, Zubrick S, Koekemoer R, Giles-Corti B. Dog walking is associated with more outdoor play and independent mobility for children. Prev Med. 2014;67:259.

11. Christian H, Francis M, Cunneen R, Zubrick S. Pets are associated with fewer peer problems and emotional symptoms, and better prosocial behavior: findings from the longitudinal study of Australian children. 220. 200-206.e2. J Pediatr. 2020:220:200-6 e2.

12. Mathers M, Canterford L, Olds T. al. e. Pet ownership and adolescent health: a cross-sectional population study. J Paediatr Child Health. 2010;46:729-35.

13. Murray JK, Browne WJ, Roberts MA, Whitmarsh A, Gruffydd-Jones TJ. Number and ownership profiles of cats and dogs in the UK. Vet Rec. 2010; 166(6):163-8.
14. Westgarth C, Pinchbeck GL, Bradshaw JW, Dawson S, Gaskell RM, Christley RM. Factors associated with dog ownership and contact with dogs in a UK community. BMC Vet Res. 2007;3(1):5.

15. Westgarth C, Boddy L, Stratton G, et al. A cross-sectional study of frequency and factors associated with dog walking in 9-10 year old children in Liverpool. UK BMC Public Health. 2013;13:822

16. Purewal R, Christley R, Kordas K, Joinson C, Meints K, Gee N, et al. Companion animals and child/adolescent development: a systematic review of the evidence. Int J Environ Res Public Health. 2017;14(3):234

17. Van Houtte BA, Jarvis PA. The role of pets in preadolescent psychosocial development. J Appl Dev Psychol. 1995:16:463-79.

18. Vidović V, Štetić V, Bratko D. Pet ownership, type of pet and socioemotional development of school children. Anthrozoös. 1999:12:211-7.

19. Smith BT. The 'pet effect': health related aspects of companion animal ownership. Aust Fam Physician. 2012:41(6):439-42.

20. Melson GF. Child development and the human-companion animal bond. Am Behav Sci. 2003:47:31-9.

21. Payne E, Bennett PC, McGreevy PD. Current perspectives on attachment and bonding in the dog-human dyad. Psychol Res Behav Manag. 2015; 8:71-9.

22. Gadomski AM, Scribani MB, Krupa N, Jenkins P. Pet dogs and child physical activity: the role of child-dog attachment. Pediatr Obes. 2017;12:e37-40.

23. Timmons B, Naylor P, Pfeiffer K. Physical activity for preschool children- how much and how? Appl Physiol Nutr Metab. 2007;32(Suppl 2F):S122-S34.

24. Lu C, Motague B. Move to learn, learn to move: Priortizing physical activity in early childhood education programming. Early Childhood Educ J. 2016; 44:409-17.

25. Burdette $H$, Whitaker $R$. Resurrecting free play in young children looking beyond fitness and fatness to attention, affiliation, and affect. Arch Pediatr Adolesc Med. 2005;159:46-50.

26. Fogel A. Developing through relationships: origins of communication, self and culture. London: University of Chicago Press; 1993.

27. Bowlby J. Attachment and loss. Harmondsworth. UK: Penguin Books; 1969.

28. Yam P, Morrison R, Penpraze V, Westgarth C, Ward D, Mutrie N, et al. Children, parents and pets exercising together (CPET) randonmised controlled trial: study rationale, design and methods. BMC Public Health. 2012;12:208

29. Richards E, Ogata N, Cheng C. Randomized controlled theory-based, email mediated walking intervention. Clin Nurs Res. 2016;26(1):47-67.

30. Schneider KL, Murphy D, Ferrara $\mathrm{C}$, et al. An online social network to increase walking in dog owners: a randomized trial. Med Sci Sports Exerc. 2015;47:631-9.

31. Gretebeck K, Bailey T, Gretebeck R. A minimal contact diet and physical activity intervention for white-collar workers. Workplace Health Saf. 2016; 65(9):417-23.

32. Chapman J, Campbell M, Wilson C. Insights for exercise adherence from a minimal planning intervention to increase physical activity. Health Educ Behav. 2015:42(6):730-5.

33. Christian H, Bauman A, Epping JN, Levine GN, McCormack G, Rhodes RE, et al. Encouraging dog walking for health promotion and disease prevention. Am J Lifestyle Med. 2016:12(3):233-43.

34. Christian H, Maitland C, Enkel S, Trapp G, Trost S, Schipperjin J, et al. Influence of the day care, home and neighbourhood environment on young children's physical activity and health: protocol for the PLAYCE observational study. BMJ. 2016;6:e014058.

35. Christian H, Rosenberg M, Trost S, Schipperijn J, Maitland C, Trapp G, et al. A snapshot of the PLAYCE project: findings from the Western Australian PLAY spaces and environments for Children's physical activity study. Supportive childcare environments for physical activity in the early years. Perth: The University of Western Australia, School of Population and Global Health; 2018.

36. Morrison R, Reilly JJ, Penpraze V, et al. Children, parents and pets exercising together (CPET): exploratory randomised controlled trial. BMC Public Health. 2013;13(1):1096

37. Hinkley T, Salmon J, Okely A, Crawford D, Hesketh K. The HAPPY study: development and reliability of a parent survey to assess correlates of preschool children's physical activity. J Sci Med Sport. 2012;15(5):407-17.

38. Burdette $H$, Whitaker $\mathrm{R}$, Daniels $\mathrm{S}$. Parental report of outdoor playtime as a measure of physical activity in preschool-aged children. Arch Pediatr Adolesc Med. 2004:158(4):353-7. 
39. Goodman R. The strengths and difficulties questionnaire: a research note. $J$ Child Psychol Psychiatry. 1997;38(5):581-6.

40. Stone L, Otten R, Engels R, Vermulst A, Janssens J. Psychometric properties of the parent and teacher versions of the strengths and difficulties questionnaire for 4- to 12-year-Olds: a review. Clin Child Fam Psychol Rev. 2010;13(3):254-74.

41. Rosenberg M. Society and the adolescent self-image. Princeton: Princeton University Press; 1965.

42. Blascovich J, Tomaka J. Measures of self-esteem. In: Robinson J, Shaver P, L W, editors. Measures of personality and social psychological attitudes. 3rd ed. Ann Arbor: Institute for Social Research; 1993. p. 115-60.

43. Rosenberg M. Conceiving the self. Malabar: Krieger; 1986.

44. Poresky $R$. The young Children's empathy measure: reliability, validity and effects of companion animal bonding. Psychol Rep. 1990;66(3):931-6.

45. The Conduct Problems Prevention Research Group. Evaluation of the first 3 years of the fast track prevention trial with children at high risk for adolescent conduct problems. J Abnorm Child Psychol. 2002;30:19-35.

46. Achenbach TM, Edelbrock CS. Behavioral problems and competencies reported by parents of normal and disturbed children aged four through sixteen. Monogr Soc Res Child Dev. 1981;46(1):82.

47. Quay H, Peterson D. Manual for the revised behaviour problem checklist Miami: Quay \& Peterson; 1987.

48. Lochman JE, The Conduct Problems Prevention Research Group. Screening of child behavior problems for prevention programs at school entry. J Consult Clin Psychol. 1995;63:549-59.

49. Cutt HE, Giles-Corti B, Knuiman MW, Pikora TJ. Physical activity behavior of dog owners: development and reliability of the dogs and physical activity (DAPA) tool. J Phys Act Health. 2008;5(Suppl 1):S73-89.

50. Aron A, Aron EN, Smollan D. Inclusion of other in the self scale and the structure of interpersonal closeness. J Pers Soc Psychol. 1992;63(4):596-612.

51. McConnell A, Brown C, Shoda T, Stayton L, Martin C. Friends with benefits: on the positive consequences of pet ownership. Am Psychol Assoc. 2011; 101(6):1239-52.

52. Cameron L, Rutland A, Brown R, Douch R. Changing children's intergroup attitudes toward refugees: testing different models of extended contact. Child Dev. 2006;77(5):1208-19.

53. Australian Government Department of Social Services. Growing up in Australia: the longitudinal study of Australian children survey. Australia: Australian Government Department of Social Services; 2005.

54. Vaughn AHD, Ward D. Measuring the physical activity practices used by parents of preschool children. Med Sci Sports Exerc. 2013;45(12):2369-77.

55. Australian Government Bureau of Meteorology. Climate Summaries Archive: Australian Government Bureau of Meteorology; [Available from: http://www. bom.gov.au/climate/current/statement_archives.shtml.

56. Engelberg JK, Carlson JA, Conway T, Cain K, Saelens B, Glanz K, et al. Dog walking among adolescents: correlates and contribution to physical activity. Prev Med. 2016;82:65-72.

57. Sirard J, Patnode C, Hearst M, Laska M. Dog ownership and adolescent physical activity. Am J Prev Med. 2011;40:334-7.

58. Christian H, Trapp G, Lauritsen C, Wright K. B G-C. understanding the relationship between dog ownership and children's physical activity and sedentary behaviour. Pediatr Obes. 2013;8:392-403.

59. Westgarth C, Ness A, Mattocks C, Christley R. A birth cohort analysis to study dog walking inAdolescence shows no relationship with objectively measured physical activity. Front Vet Sci. 2017;4:62.

60. Westgarth C, Boddy L, Stratton G, German A, Gaskell R, Coyne K, et al. A cross-sectional study of frequency and factors associated with dog walking in 9-10 year old children in Liverpool, UK. BMC Public Health. 2013;13:822.

61. Wenden $\mathrm{E}$, Lester L, Zubrick S, Ng M, Christian $\mathrm{H}$. The relationship between dog ownership, dog play, family dog walking and preschooler socialemotional development: findings from the PLAYCE observational study. Pediatr Res. 2020.

62. Paul E, Serpell J. Obtaining a new pet dog: effects on middle childhood children and their families. Appl Anim Behav Sci. 1996;47:17-29.

63. Morgan WP. Prescription of physical activity: a paradigm shift. Quest. 2001; 53:366-82.

64. Rhodes R, Murray H, Temple V, Tuokko H, Higgins J. Pilot study of a dog walking randomized intervention: effects of a focus on canine exercise. Prev Med. 2012;34:309-12.

\section{Publisher's Note}

Springer Nature remains neutral with regard to jurisdictional claims in published maps and institutional affiliations.
Ready to submit your research? Choose BMC and benefit from:

- fast, convenient online submission

- thorough peer review by experienced researchers in your field

- rapid publication on acceptance

- support for research data, including large and complex data types

- gold Open Access which fosters wider collaboration and increased citations

- maximum visibility for your research: over $100 \mathrm{M}$ website views per year

At BMC, research is always in progress.

Learn more biomedcentral.com/submissions 